\title{
SENSORS FAULT DIAGNOSIS ALGORITHM DESIGN OF A HYDRAULIC SYSTEM
}

\author{
Matej ORAVEC, Anna JADLOVSKÁ
}

Department of Cybernetics and Artificial Intelligence, Faculty of Electrical Engineering and Informatics, Technical University of Košice, Letná 9, 04200 Košice, Slovak Republic, Tel.: +421 55602 4214, E-mail: matej.oravec@tuke.sk, anna.jadlovska@tuke.sk

\begin{abstract}
This article presents the sensors fault diagnosis system design for the hydraulic system, which is based on the group of the three fault estimation filters. These filters are used for estimation of the system states and sensors fault magnitude. Also, this article briefly stated the hydraulic system state control design with integrator, which is important assumption for the fault diagnosis system design. The sensors fault diagnosis system is implemented into the Matlab/Simulink environment and it is verified using the controlled hydraulic system simulation model. Verification of the designed fault diagnosis system is realized by series of experiments, which simulates sensors faults. The results of the experiments are briefly presented in the last part of this article.
\end{abstract}

Keywords: sensor fault, fault diagnosis system, fault estimation, hydraulic system

\section{INTRODUCTION}

Growing complexity of the controlled systems leads to increasing chance of the system failure. The faults of the system have negative influence to its control. The system actuators and sensors are more sensible to the fault occurrence. At present, a lot of attention is paid to diagnosis system design, which are used for actuators or sensors fault detection, isolation and estimation.

Different fault diagnosis methods are used for the diagnosis system design. The selected fault diagnosis methods, based on the model of the system, are presented in books [1], [2] and publications [7], [8]. In publication [4] authors present sensors fault diagnosis method based on principles of Kalman filtering and they use this method for fault diagnosis of the time-varying system. In [3], the authors present the diagnosis system and fault tolerant control design, which is verified using the controlled three tank hydraulic system. Different approaches of the diagnosis system design are listed in [5], [6] for the various types of the hydraulic systems.

This paper is dedicated to design of algorithms for the diagnosis of the hydraulic system sensors fault, which are based on method presented in [4]. Also, this paper stated the algorithms implementation to the Matlab/Simulink environment. Designed algorithms are used for the detection, localization and estimation of the hydraulic system sensors fault. The control of the hydraulic system is very important step to proper functionality of the diagnosis system.

The second section of this paper is dedicated to algorithm design for the hydraulic system state control with the integrator. The third section is focused on the sensors fault diagnosis algorithms design for the hydraulic system. The designed control algorithm and functionality of the diagnosis system is verified by experiments and shortly discussed in the fourth section of this paper.

Sensors fault diagnosis algorithms design for the hydraulic system is related to the tasks solved within the project "University Science Park TECHNICOM for Innovation Applications Supported by Knowledge Technology" with subactivity "Center for Nondestructive Diagnostics of Technological Processes".

\section{MODELLING AND STATE CONTROL DESIGN OF THE HYDRAULIC SYSTEM}

Considered hydraulic system consists of the three identical cylindrical tanks in interaction. Flow-rates $q_{1}(t), q_{2}(t)$ are inputs to the first and second tank. Liquid leaks out of the second tank with flow-rate $q_{20}(t)$. Outputs of the hydraulic system are liquid levels in tanks, which are denoted $h_{1}(t), h_{2}(t), h_{3}(t)$ [1]. Schematic structure of the hydraulic system is illustrated in Fig. 1. Denotation of the hydraulic system physical variables and parameters is listed in Table 1.

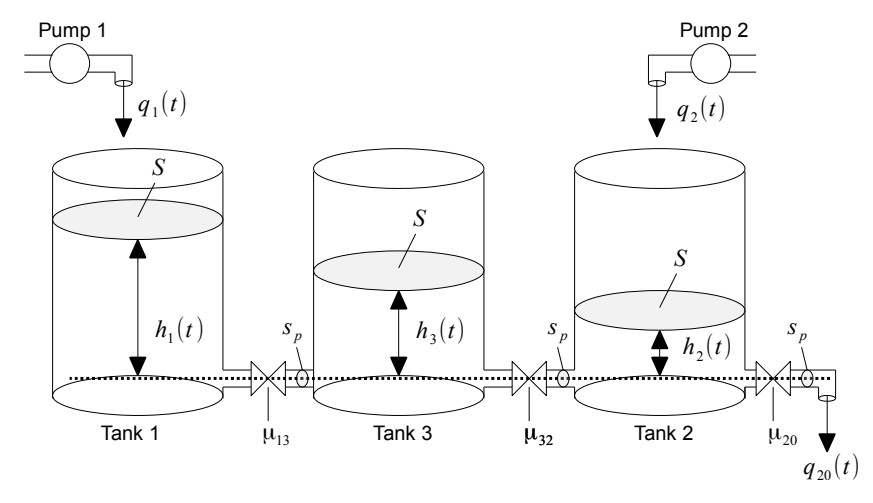

Fig. 1 Hydraulic system schematic representation 
Table 1 Denotation of hydraulic system variables and parameters

\begin{tabular}{|ll|}
\hline inter tank cross sectional area & $s_{p}\left[\mathrm{~m}^{2}\right]$ \\
1st tank outflow coefficient & $\mu_{13}[\%]$ \\
3rd tank outflow coefficient & $\mu_{32}[\%]$ \\
2nd tank outflow coefficient & $\mu_{20}[\%]$ \\
\hline 1st tank input flow rate & $q_{1}(t)\left[\mathrm{m}^{3} / \mathrm{s}\right]$ \\
2nd tank input flow rate & $q_{2}(t)\left[\mathrm{m}^{3} / \mathrm{s}\right]$ \\
2nd tank output flow rate & $q_{20}(t)\left[\mathrm{m}^{3} / \mathrm{s}\right]$ \\
1st tank level & $h_{1}(t)[\mathrm{m}]$ \\
2nd tank level & $h_{2}(t)[\mathrm{m}]$ \\
3rd tank level & $h_{3}(t)[\mathrm{m}]$ \\
\hline maximum flow rate $q_{1 \max }, q_{2 \max }$ & $1.5 \times 10^{-4} \mathrm{~m}^{3} \mathrm{~s}^{-1}$ \\
maximum level $h_{1 \max }, h_{2 \max }, h_{2 \max }$ & $0.62 \mathrm{~m}$ \\
\hline
\end{tabular}

Modelling and control design of the hydraulic system is important assumption for the fault diagnosis. Controlled hydraulic system without fault occurrence is considered as nominal system.

\subsection{Modelling of hydraulic system}

According to the hydraulic system schematic representation (Fig. 1) and mass balance equations, the mathematical model of the hydraulic system is composed in form of the differential equations as follows:

$$
\begin{aligned}
& \frac{d h_{1}(t)}{d t}=\frac{1}{S}\left(q_{1}(t)-q_{13}(t)\right), \\
& \frac{d h_{2}(t)}{d t}=\frac{1}{S}\left(q_{2}(t)+q_{32}(t)-q_{20}(t)\right), \\
& \frac{d h_{3}(t)}{d t}=\frac{1}{S}\left(q_{13}(t)-q_{32}(t)\right),
\end{aligned}
$$

where water flow-rates $q_{13}(t), q_{32}(t), q_{20}(t)$ between tanks are determined using the Torriceli - rule:

$$
\begin{aligned}
& q_{13}(t)=\underbrace{\mu_{13} s_{p} \sqrt{2 g}}_{k_{13}} \sqrt{\left|h_{1}(t)-h_{3}(t)\right|}, \\
& q_{32}(t)=\underbrace{\mu_{32} s_{p} \sqrt{2 g}}_{k_{32}} \sqrt{\left|h_{3}(t)-h_{2}(t)\right|}, \\
& q_{20}(t)=\underbrace{\mu_{20} s_{p} \sqrt{2 g}}_{k_{20}} \sqrt{h_{2}(t)},
\end{aligned}
$$

under assumption $h_{1}(t)>h_{3}(t)>h_{2}(t)$.

Mathematical model of the hydraulic system (1) can be expressed using the nonlinear state-space description:

$\dot{\mathbf{x}}(t)=\mathbf{f}(\mathbf{x}(t), \mathbf{u}(t), t)$,

$\mathbf{y}(t)=\mathbf{g}(\mathbf{x}(t), t)$,

with the state vector $\mathbf{x}(t) \in \mathbb{R}^{n}$ :

$\mathbf{x}(t)=\left[x_{1}(t), x_{2}(t), x_{3}(t)\right]^{T}=\left[h_{1}(t), h_{2}(t), h_{3}(t)\right]^{T}$,

the input vector $\mathbf{u}(t) \in \mathbb{R}^{p}$ :

$\mathbf{u}(t)=\left[u_{1}(t), u_{2}(t)\right]^{T}=\left[q_{1}(t), q_{2}(t)\right]^{T}$ and the output vector $\mathbf{y}(t) \in \mathbb{R}^{m}$ :

$\mathbf{y}(t)=\left[h_{1}(t), h_{2}(t), h_{3}(t)\right]^{T}$

The general nonlinear state-space description (3) of the hydraulic system can be rewritten in form:

$$
\begin{aligned}
& \dot{x}_{1}(t)=\underbrace{\frac{1}{S}\left(u_{1}(t)-k_{13} \sqrt{\left|x_{1}(t)-x_{3}(t)\right|}\right)}_{f_{1}(\mathbf{x}(t), \mathbf{u}(t), t)} \\
& \dot{x}_{2}(t)=\underbrace{\frac{1}{S}\left(u_{2}(t)-k_{20} \sqrt{x_{2}(t)}+k_{32} \sqrt{\left|h_{3}(t)-h_{2}(t)\right|}\right)}_{f_{2}(\mathbf{x}(t), \mathbf{u}(t), t)} \\
& \dot{x}_{3}(t)=\underbrace{\frac{1}{S}\left(k_{13} \sqrt{\left|x_{1}(t)-x_{3}(t)\right|}-k_{32} \sqrt{\left|x_{3}(t)-x_{2}(t)\right|}\right)}_{f_{3}(\mathbf{x}(t), \mathbf{u}(t), t)} \\
& \mathbf{y}(t)=\underbrace{\mathbf{I}_{3} \mathbf{x}(t)}_{\mathbf{g}(\mathbf{x}(t), t)}
\end{aligned}
$$

The nonlinear state-space description (7) of the hydraulic system is linearized around the operating point $\mathbf{O P}=\left[\mathbf{x}^{o p}, \mathbf{u}^{o p}\right]$ using the Taylor expansion and subsequently discretized with the sampling period $T_{s}=1 \mathrm{~s}$ for the state control design purpose. The result of the nonlinear state-space description (7) linearization and discretization is the linear deviation model of the hydraulic system in discrete state-space form:

$$
\begin{aligned}
& \Delta \mathbf{x}(k+1)=\mathbf{A}_{d} \Delta \mathbf{x}(k)+\mathbf{B}_{d} \Delta \mathbf{u}(k), \\
& \Delta \mathbf{y}(k)=\mathbf{C} \Delta \mathbf{x}(k),
\end{aligned}
$$

where $\mathbf{A}_{d} \in \mathbb{R}^{n \times n}, \mathbf{B}_{d} \in \mathbb{R}^{n \times p}$ and $\mathbf{C} \in \mathbb{R}^{m \times n}$ represents the system dynamic, input and output matrices.

Discrete state space model (8) of the hydraulic system is used for the state control algorithm design with integrator of the control error.

\subsection{State Control Design of Hydraulic System}

The state control with integrator of the control error $\mathbf{z}(k)$ is used for the tracking of the step changes of the reference tanks levels. To fulfil the condition of controllability, only the first and second tank of the hydraulic system are controlled [3]. Under this assumption, the output equation of the discrete state space model (8) of the hydraulic system can be rewritten to the form:

$\Delta \mathbf{y}(k)=\mathbf{C} \Delta \mathbf{x}(k)=\left[\begin{array}{l}\mathbf{C}_{1} \\ \mathbf{C}_{2}\end{array}\right] \Delta \mathbf{x}(k)$,

where matrix $\mathbf{C}_{1}=\left[\begin{array}{lll}1 & 0 & 0 \\ 0 & 1 & 0\end{array}\right]$ and matrix $\mathbf{C}_{2}=\left[\begin{array}{lll}0 & 0 & 1\end{array}\right]$.

The control objective is to ensure that:

$\lim _{k \rightarrow \infty} \mathbf{C}_{1} \Delta \mathbf{x}(k)=\mathbf{y}_{\text {ref }}(k)$

To achieve this task, the discrete-time integrator of the control error $\mathbf{z}(k)$ is added to the state control design, which is computed:

$\mathbf{z}(k+1)=\mathbf{z}(k)+T_{s}\left(\mathbf{y}_{r e f}(k)-\mathbf{C}_{1} \Delta \mathbf{x}(k)\right)$ 
According to the system output (9) and control error (11) expressions, the augmented state-space form of the hydraulic system linear model can be determined:

$$
\begin{aligned}
{\left[\begin{array}{c}
\Delta \mathbf{x}(k+1) \\
\mathbf{z}(k+1)
\end{array}\right] } & =\mathbf{A}_{c}\left[\begin{array}{c}
\Delta \mathbf{x}(k) \\
\mathbf{z}(k)
\end{array}\right]+\mathbf{B}_{c} \Delta \mathbf{u}(k)+ \\
& +\mathbf{I}_{c} \mathbf{y}_{r e f}(k), \\
\Delta \mathbf{y}(k) & =\mathbf{C}_{c}\left[\begin{array}{c}
\Delta \mathbf{x}(k) \\
\mathbf{z}(k)
\end{array}\right],
\end{aligned}
$$

with

$$
\begin{aligned}
& \mathbf{A}_{c}=\left[\begin{array}{cc}
\mathbf{A}_{d} & \mathbf{0} \\
-T_{s} \mathbf{C}_{1} & \mathbf{I}
\end{array}\right], \mathbf{B}_{c}=\left[\begin{array}{c}
\mathbf{B}_{d} \\
\mathbf{0}
\end{array}\right], \\
& \mathbf{I}_{c}=\left[\begin{array}{c}
\mathbf{0} \\
T_{s} \mathbf{I}_{2}
\end{array}\right], \mathbf{C}_{c}=\left[\begin{array}{ll}
\mathbf{C}
\end{array}\right]
\end{aligned}
$$

This augmented state-space representation is used for the control design using the pole placement method. The state control law with the integrator $\mathbf{z}(k)$ has form:

$\Delta \mathbf{u}(k)=-\mathbf{K}\left[\begin{array}{c}\Delta \mathbf{x}(k) \\ \mathbf{z}(k)\end{array}\right]$,

where feedback matrix $\mathbf{K}$ can be divided into the two matrices $\mathbf{K}_{1}, \mathbf{K}_{2}$ and control law (14) can be expressed:

$$
\Delta \mathbf{u}(k)=-\mathbf{K}_{1} \Delta \mathbf{x}(k)-\mathbf{K}_{2} \mathbf{z}(k)
$$

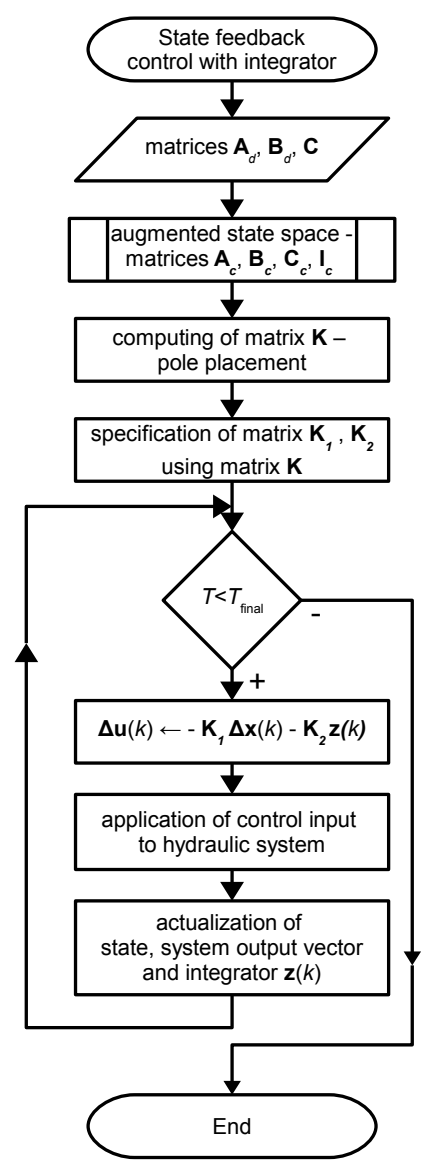

Fig. 2 State feedback control with integrator flowchart

The results obtained by designed control algorithm for the hydraulic system are illustrated in Fig. 4, in form of the time responses of the tanks levels $h_{1}(t), h_{2}(t), h_{3}(t)$ and input flow rates $q_{1}(t), q_{2}(t)$.

The algorithm of the state control with integrator is designed (Fig. 2) and implemented in Matlab/Simulink environment (Fig. 3).

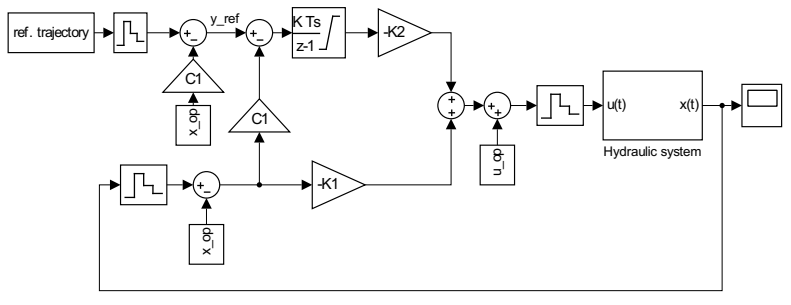

Fig. 3 State feedback control structure with integrator
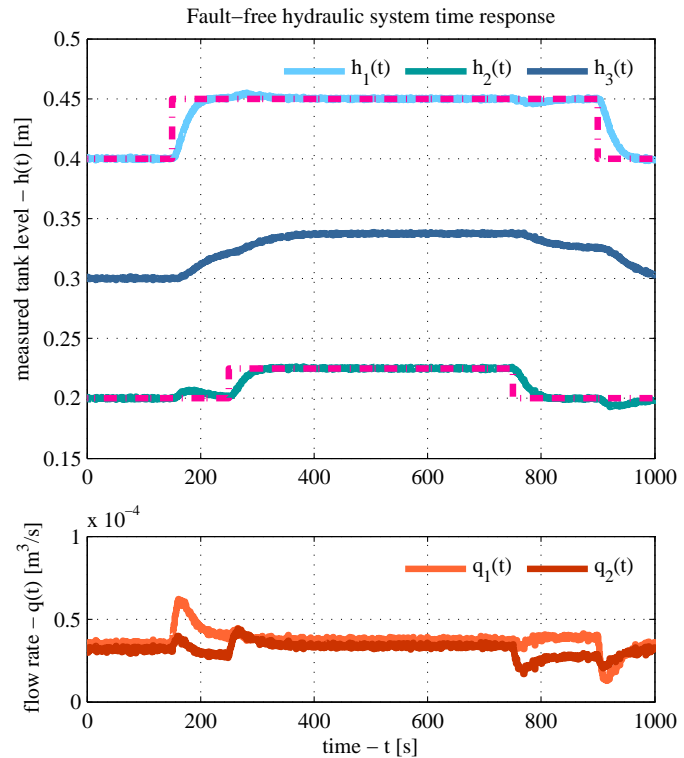

Fig. 4 Hydraulic system control simulation - nominal system

The results of control of the nominal system are used for the evaluation of the generated residuals in sensors fault detection of the hydraulic system.

\section{SENSORS FAULT DIAGNOSIS SYSTEM}

The design of sensors fault diagnosis algorithms is important step to increase reliability of the hydraulic system control. Algorithms of the sensors fault diagnosis are implemented into the complex diagnosis system. Concept of the considered diagnosis system is composed from the fault detection, isolation and estimation part (Fig. 5).

The fault diagnosis system is based on group of the three fault estimation filters and each fault estimation filter is implemented in Matlab/Simulink environment according to the designed algorithm. These filters are used for the system states and fault magnitude estimation. Estimation of the system states is very important for the fault detection. Each filter is insensible to fault of one of the three sensors what is used for the fault isolation. 


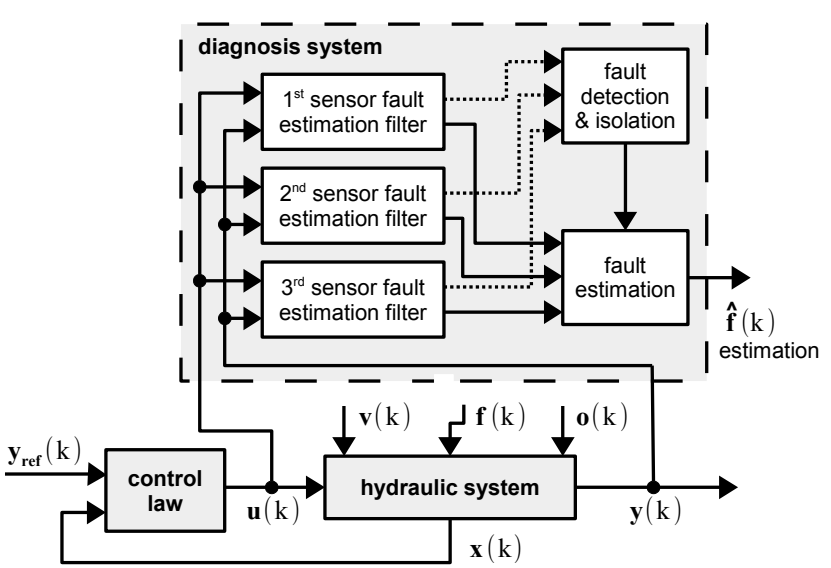

Fig. 5 Concept scheme of the sensors fault diagnosis system

\subsection{Sensor Fault Estimation Filter Design}

This subsection presents algorithm design of the filter for the sensor fault estimation, which is based on the fault diagnosis method listed in [4].

The fault estimation filter design is based on the faulty model of the hydraulic system, which is derived using the discrete state space model of the system (8). Model of the hydraulic system with fault has form:

$\Delta \mathbf{x}(k+1)=\mathbf{A}_{d} \Delta \mathbf{x}(k)+\mathbf{B}_{d} \Delta \mathbf{u}(k)+\mathbf{v}(k)$

$\Delta \mathbf{y}(k)=\mathbf{C} \Delta \mathbf{x}(k)+\mathbf{F}_{s} \mathbf{f}(k)+\mathbf{o}(k)$

where discrete state space model of the system $(8)$ is expanded by the sensors fault matrix $\mathbf{F}_{s} \in \mathbb{R}^{m \times q}$. The vector $\mathbf{f}(k) \in \mathbb{R}^{q}$ consists of magnitude of the sensors fault.

It is considered that the system noise $\mathbf{v}(k) \in \mathbb{R}^{n}$ and measurement noise $\mathbf{o}(k) \in \mathbb{R}^{m}$ are uncorrelated white noises with covariance matrices $\mathbf{Q}=E\left\{\mathbf{v}(k) \mathbf{v}^{T}(k)\right\} \geq 0$, $\mathbf{R}=E\left\{\mathbf{o}(k) \mathbf{o}^{T}(k)\right\}>0$, respectively.

Under assumption presented in [4] that fault of all sensors is very unlikely at the same time, the matrix $\mathbf{F}_{s}$ have to satisfy these two conditions:

1. $\operatorname{rank}\left(\mathbf{F}_{s}\right)=q, q<m$,

2. $\operatorname{rank}\left(\mathbf{C F}_{s}\right)=\operatorname{rank}\left(\mathbf{F}_{s}\right)$,

where $m$ denotes number of the system outputs.

For design of the three sensors fault estimation filters, it is considered that matrix $\mathbf{F}_{s} \in\left\{\mathbf{F}_{s 1}, \mathbf{F}_{s 2}, \mathbf{F}_{s 3}\right\}$. According to the assumptions (17) for the sensors fault matrix $\mathbf{F}_{s}$ using the system outputs matrix $\mathbf{C}$ form, it can be expressed fault matrix for each sensor $\mathbf{F}_{s 1}=[1,0,0]^{T}, \mathbf{F}_{s 2}=[0,1,0]^{T}$, $\mathbf{F}_{s 3}=[0,0,1]^{T}$.

Model of the hydraulic system with fault 16 can be written, according to [4], in the descriptor form:

$$
\begin{aligned}
& \mathbf{E} \tilde{\mathbf{x}}(k+1)=\tilde{\mathbf{A}} \tilde{\mathbf{x}}(k)+\tilde{\mathbf{B}} \Delta \mathbf{u}(k)+\tilde{\mathbf{G}} \mathbf{v}(k) \\
& \Delta \mathbf{y}(k)=\tilde{\mathbf{C}} \tilde{\mathbf{x}}(k)+\mathbf{o}(k)
\end{aligned}
$$

where:

$$
\begin{aligned}
& \tilde{\mathbf{x}}(k)=\left[\begin{array}{c}
\Delta \mathbf{x}(k) \\
\mathbf{f}(k)
\end{array}\right], \mathbf{E}=\left[\begin{array}{cc}
\mathbf{I}_{n} & \mathbf{0} \\
\mathbf{0} & \mathbf{0}
\end{array}\right], \\
& \tilde{\mathbf{A}}=\left[\begin{array}{cc}
\mathbf{A}_{d} & \mathbf{0} \\
\mathbf{0} & \mathbf{0}
\end{array}\right], \tilde{\mathbf{B}}=\left[\begin{array}{c}
\mathbf{B}_{d} \\
\mathbf{0}
\end{array}\right], \\
& \tilde{\mathbf{G}}=\left[\begin{array}{c}
\mathbf{I}_{n} \\
\mathbf{0}
\end{array}\right], \tilde{\mathbf{C}}=\left[\begin{array}{ll}
\mathbf{C} & \mathbf{F}_{s}
\end{array}\right]
\end{aligned}
$$

and $\mathbf{I}_{n}$ represents identity matrix of $n \times n$ size.

For the estimation of the state vector $\tilde{\mathbf{x}}(k)$ of the hydraulic system in descriptor form (18), it is possible to design filter using principles of Kalman filtering in form:

$$
\begin{aligned}
\hat{\tilde{\mathbf{x}}}(k+1 \mid k) & =\mathbf{T} \tilde{\mathbf{A}} \hat{\tilde{\mathbf{x}}}(k \mid k-1)+\mathbf{T} \tilde{\mathbf{B}} \Delta \mathbf{u}(k)+ \\
& +\mathbf{L}(k)(\Delta \mathbf{y}(k)-\tilde{\mathbf{C}} \hat{\tilde{\mathbf{x}}}(k \mid k-1))+ \\
& +\mathbf{N} \Delta \mathbf{y}(k+1),
\end{aligned}
$$

where matrices $\mathbf{T} \in \mathbb{R}^{(n+q) \times(n+q)}, \mathbf{N} \in \mathbb{R}^{(n+q) \times m}, \mathbf{L} \in$ $\mathbb{R}^{(n+q) \times m}$. Matrices $\mathbf{T}, \mathbf{N}$ have to be designed under assumption:

$\mathbf{T E}+\mathbf{N} \tilde{\mathbf{C}}=\mathbf{I}_{n+q}$

and these matrices are given by relations:

$$
\begin{aligned}
& \mathbf{T}=\Phi^{+} \phi_{1}+\mathbf{I}_{\varphi}\left(\mathbf{I}_{n+q+m}-\Phi \Phi^{+}\right) \phi_{1}, \\
& \mathbf{N}=\Phi^{+} \phi_{2}+\mathbf{I}_{\varphi}\left(\mathbf{I}_{n+q+m}-\Phi \Phi^{+}\right) \phi_{2},
\end{aligned}
$$

with:

$\Phi=\left[\begin{array}{c}\mathbf{E} \\ \tilde{\mathbf{C}}\end{array}\right], \phi_{1}=\left[\begin{array}{c}\mathbf{I}_{n+q} \\ \mathbf{0}\end{array}\right], \phi_{2}=\left[\begin{array}{c}\mathbf{0} \\ \mathbf{I}_{m}\end{array}\right], \mathbf{I}_{\varphi}=\left[\begin{array}{ll}\mathbf{I}_{n+q} & \mathbf{0}\end{array}\right]$,

where $\Phi^{+}$denotes pseudoinverse of matrix $\Phi$

The gain matrix $\mathbf{L}(k)$ of the fault estimation filter 20) minimizes the trace of error covariance matrix $\mathbf{P}(k \mid k-1)=$ $E\left\{(\tilde{\mathbf{x}}(k)-\hat{\tilde{\mathbf{x}}}(k \mid k-1))(\tilde{\mathbf{x}}(k)-\hat{\tilde{\mathbf{x}}}(k \mid k-1))^{T}\right\}$ and it is computed in each $k$ step:

$$
\mathbf{L}(k)=\mathbf{T} \tilde{\mathbf{A}} \mathbf{P}(k \mid k-1) \tilde{\mathbf{C}}^{T}\left[\tilde{\mathbf{C}} \mathbf{P}(k \mid k-1) \tilde{\mathbf{C}}^{T}+\mathbf{R}\right]^{-1}
$$

where estimation error covariance matrix $\mathbf{P}(k \mid k-1)$ is computed by iterative procedure:

$$
\begin{aligned}
\mathbf{P}(k+1 \mid k) & =\mathbf{T} \tilde{\mathbf{A}} \mathbf{P}(k \mid k-1)(\mathbf{T} \tilde{\mathbf{A}})^{T}- \\
& -\mathbf{L}(k) \tilde{\mathbf{C}} \mathbf{P}(k \mid k-1)(\mathbf{T} \tilde{\mathbf{A}})^{T}+ \\
& +\mathbf{T} \tilde{\mathbf{G}} \mathbf{Q}(\mathbf{T} \tilde{\mathbf{G}})^{T}+\mathbf{N R} \mathbf{N}^{T}
\end{aligned}
$$

The algorithm for the estimation of hydraulic system states and fault of the selected sensor is designed using expressions presented in this subsection. The designed algorithm is illustrated as flowchart in Fig. 7. The computation of the $\mathbf{T}, \mathbf{N}$ matrices expressed in 22 is implemented as $k f e$ paramA function. Inputs for the $k f e$ param $A$ function are matrices of the faulty model and outputs are matrices of the faulty model in descriptor form (18) with matrices $\mathbf{T}, \mathbf{N}$. Computation of the gain matrix $\mathbf{L}(k)$ and covariance matrix $\mathbf{P}(k+1 \mid k)$ is implemented as Matlab function called FE_Kalman_Gain.

Filter equation expressed in 20 is implemented in Simulink environment and its simulation scheme is illustrated in Fig. 6 Estimation of the hydraulic system states by created filter based on designed algorithm is used for the hydraulic system sensors fault detection and isolation. 


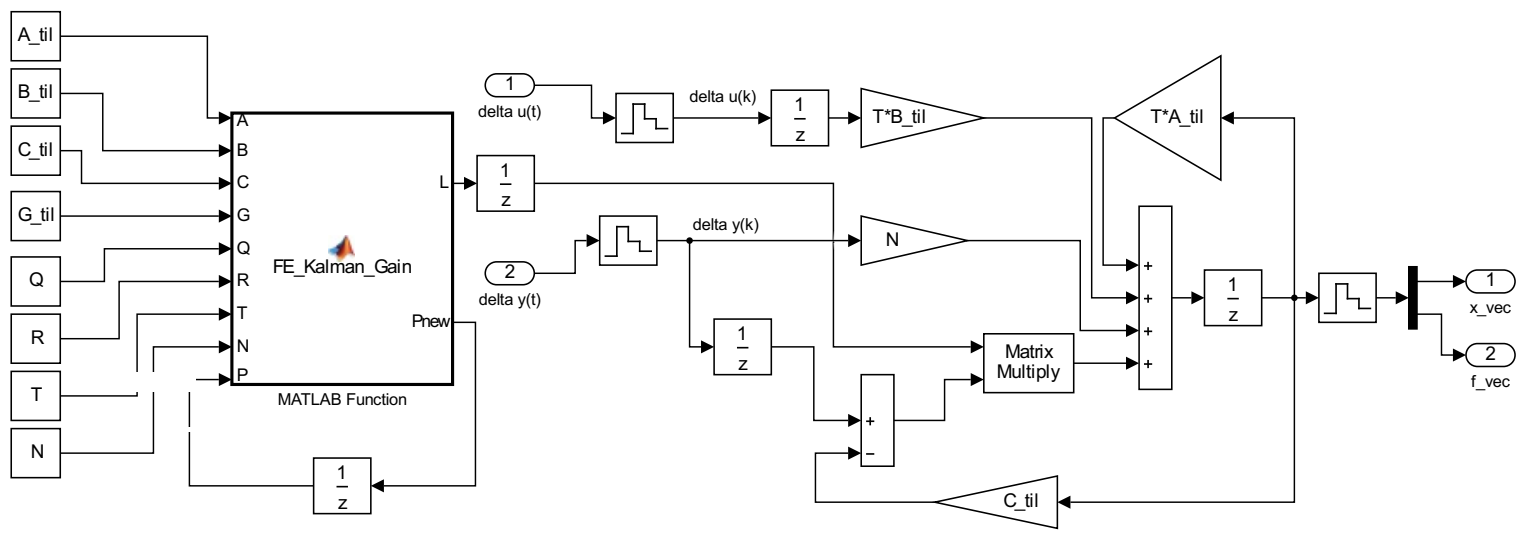

Fig. 6 Fault estimation filter implemented in Simulink

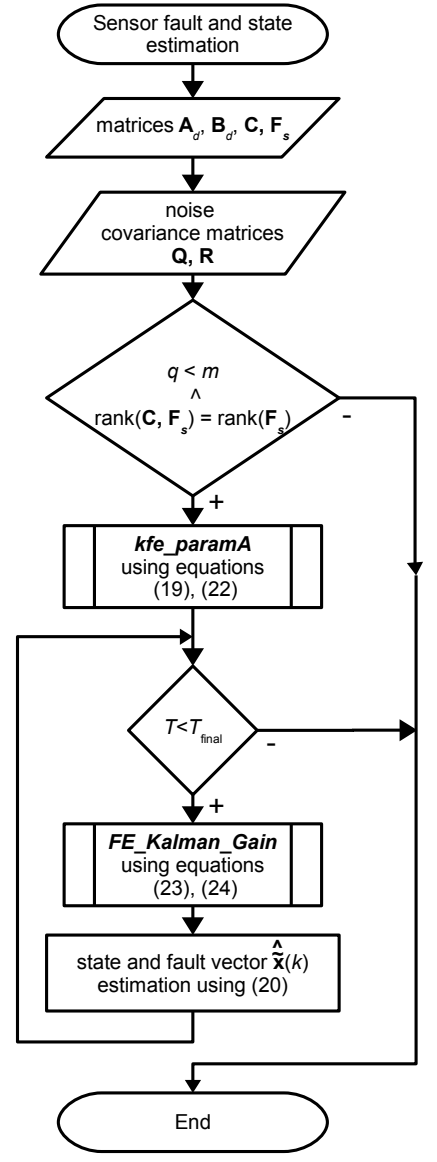

Fig. 7 Selected sensor fault and hydraulic system states estimation algorithm

\subsection{Sensor Fault Detection and Isolation}

Fault detection and isolation methods are based on residual generation and evaluation in each simulation step. If generated residual exceeds the threshold value, then diagnosis system generates symptom and produces information about fault occurrence. Generated symptom is used for localization of fault occurrence [2].

Sensor fault detection uses residual generation in form:

$\mathbf{r}(k)=\Delta \mathbf{y}(k)-\tilde{\mathbf{C}} \hat{\tilde{\mathbf{x}}}(k \mid k-1)$, where matrix $\tilde{\mathbf{C}}=\left[\mathbf{C}, \mathbf{F}_{s}\right]$. As it is noted in subsection 3.1 matrix $\mathbf{F}_{s} \in\left\{\mathbf{F}_{s 1}, \mathbf{F}_{s 2}, \mathbf{F}_{s 3}\right\}$. Under this assumption, the designed sensors fault diagnosis system contains three generators of the residuals $\mathbf{r}_{1}, \mathbf{r}_{2}, \mathbf{r}_{3}[4]$.

The residuals evaluation is based on approach presented in [2] with using the RMS values as follows:

$\left\|\mathbf{r}_{i}(k)\right\|_{R M S}=\sqrt{\frac{1}{N} \sum_{j=k-N+1}^{k}\left(\mathbf{r}_{i}(j) \mathbf{r}_{i}^{T}(j)\right)}, i=1,2,3$

It is important to determine residual threshold values using the nominal system simulation results for their evaluation:

$\tau_{i}=\sup _{\text {fault }- \text { free }}\left\|\mathbf{r}_{i}(k)\right\|_{R M S}, i=1,2,3$

Symptoms $s_{i}$ for $i=1,2,3$, are generated according to evaluation conditions:

$$
\begin{gathered}
\left\|\mathbf{r}_{i}(k)\right\|_{R M S}>\tau_{i}, \text { then } s_{i}=1 \\
\left\|\mathbf{r}_{i}(k)\right\|_{R M S} \leq \tau_{i}, \text { then } s_{i}=0 .
\end{gathered}
$$

Combinations of the selected symptoms $s_{i}$ are listed in Table 2 and they are used for the fault isolation.

The three sensor fault estimation filters are implemented in Simulink (Fig. 8) and each filter is designed according to the algorithm presented in Fig. 7. In Matlab, the m-file is created for the residuals RMS evaluation and symptoms generation what is used for the fault detection and isolation. Both presented program modules are the parts of the diagnosis system and they are verified by the simulation experiments.

Table 2 Sensors fault isolation

\begin{tabular}{|c|cccc|}
\hline & $\begin{array}{c}\text { no } \\
\text { fault }\end{array}$ & $\begin{array}{c}\text { sensor 1 } \\
\text { fault }\end{array}$ & $\begin{array}{c}\text { sensor 2 } \\
\text { fault }\end{array}$ & $\begin{array}{c}\text { sensor 3 } \\
\text { fault }\end{array}$ \\
\hline$s_{1}$ & 0 & 0 & 1 & 1 \\
$s_{2}$ & 0 & 1 & 0 & 1 \\
$s_{3}$ & 0 & 1 & 1 & 0 \\
\hline
\end{tabular}




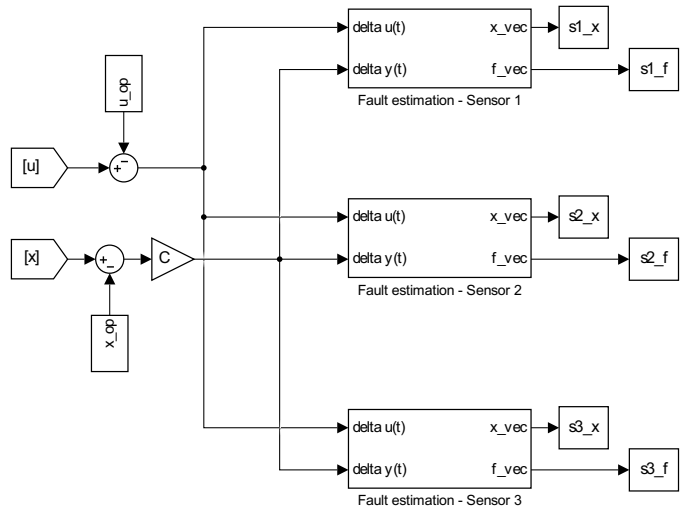

Fig. 8 Group of sensors faults estimation filters implemented in Simulink

\section{SIMULATION VERIFICATION OF SENSORS FAULT DIAGNOSIS SYSTEM}

The created sensors fault diagnosis system is verified by the four experiments using simulation model of the hydraulic system with the time duration $T_{\text {final }}=1000 \mathrm{~s}$, and the sampling period $T_{s}=1 \mathrm{~s}$. The first experiment is realized without sensors fault and results illustrates behaviour of the nominal system. The time responses of the tanks levels and the inputs of the controlled hydraulic system are illustrated in Fig. 4. The RMS values of residuals $\left\|\mathbf{r}_{1}(k)\right\|_{R M S},\left\|\mathbf{r}_{2}(k)\right\|_{R M S},\left\|\mathbf{r}_{3}(k)\right\|_{R M S}$ computed in every sample of the simulation are shown in Fig. 9 The result of this experiment is used for the determination of the residual threshold $\tau_{1}, \tau_{2}, \tau_{3}$ values.
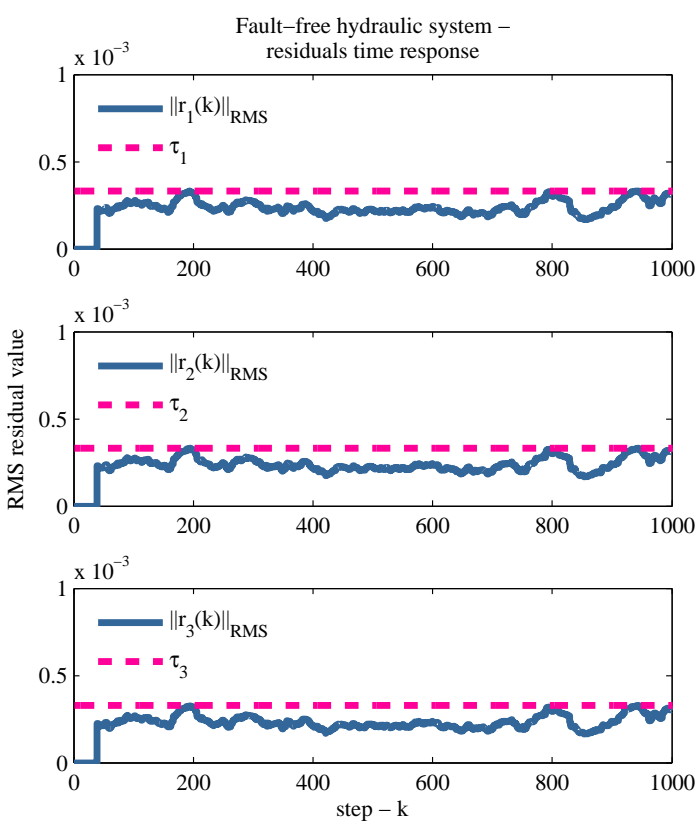

Fig. 9 Time responses of the residuals RMS values for the fault-free case

The various faults of each sensor are simulated in the next three experiments. In the second experiment is simulated abrupt sensor fault. The real and estimated time response of the sensor fault is illustrated in Fig. 10. The residuals evaluation in Fig. 11 shows that the first sensor of the hydraulic system is affected by fault. The fault of the first sensor is detected in $T=450 \mathrm{~s}$ and localized around the time $T=542 \mathrm{~s}$. The result of the hydraulic system control with the fault of the first sensor is illustrated in Fig. 12

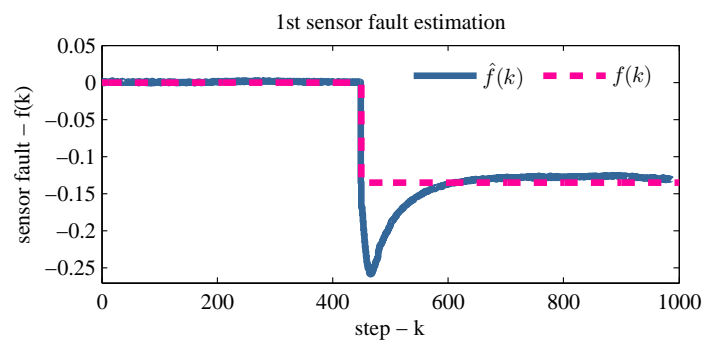

Fig. 10 Estimation of the abrupt sensor fault - the first sensor
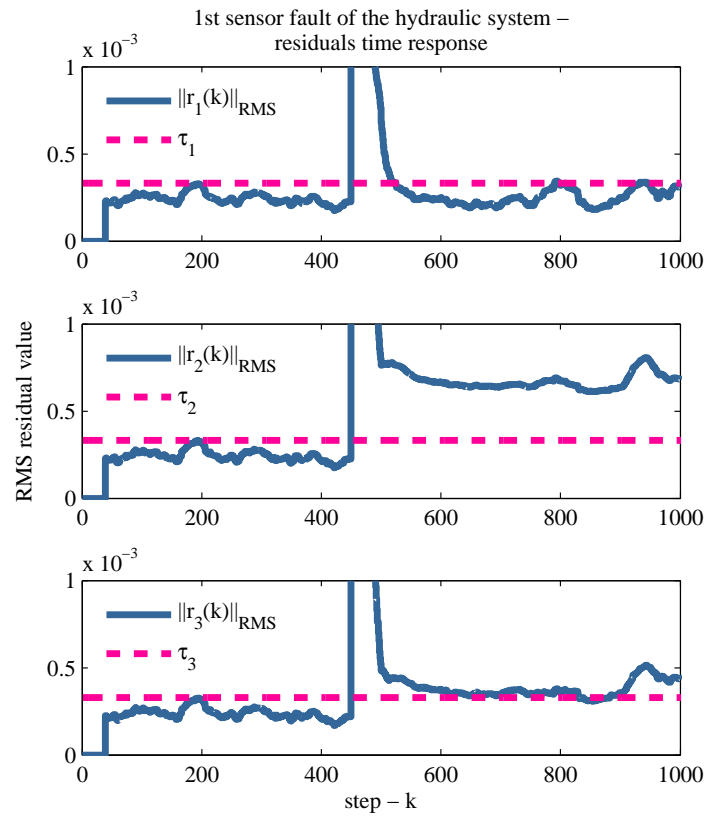

Fig. 11 Time responses of the residuals RMS values for the abrupt sensor fault - the first sensor
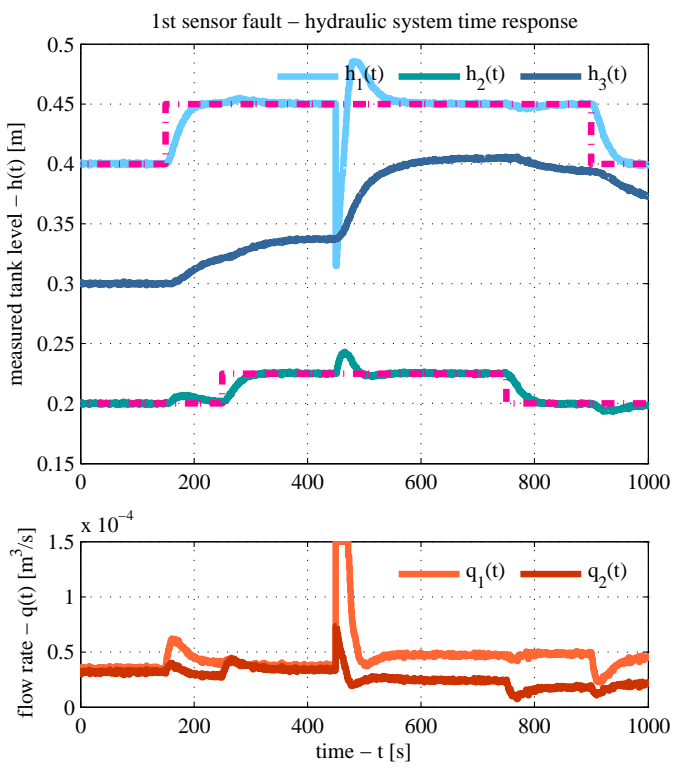

Fig. 12 Control of the hydraulic system with the first sensor fault occurrence 
Incipient fault of the sensor is simulated in the third experiment. The real and estimated fault magnitude is illustrated in Fig. 13 The time responses of the residuals RMS values (Fig. 14) show that incipient fault affects the second sensor of the hydraulic system. Fault occurrence is detected and isolated in $T=369 \mathrm{~s}$.

In the last experiment is affected the third sensor of the hydraulic system by intermittent fault. Time response of the real and estimated fault magnitude is illustrated in Fig. 15 The residuals RMS values with selected thresholds $\tau_{i}, i=1,2,3$ are shown in Fig. 16 Intermittent fault is isolated $41 \mathrm{~s}$ after its detection.

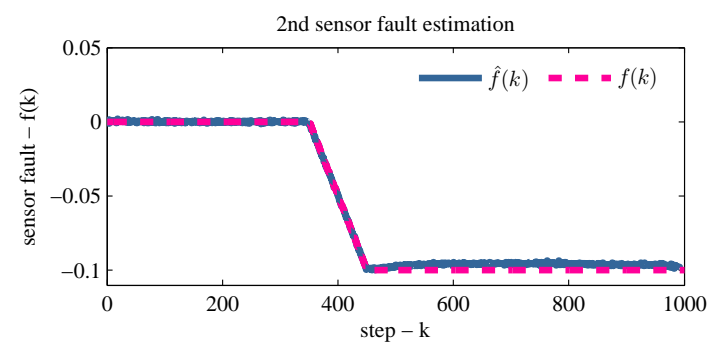

Fig. 13 Estimation of the incipient sensor fault - the second sensor
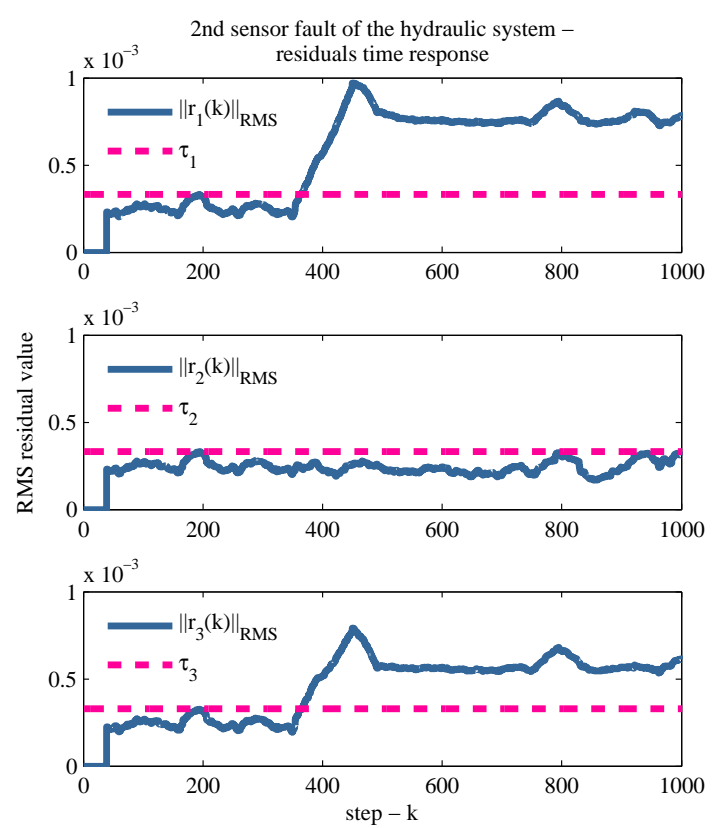

Fig. 14 Time responses of the residuals RMS values for the incipient sensor fault - the second sensor

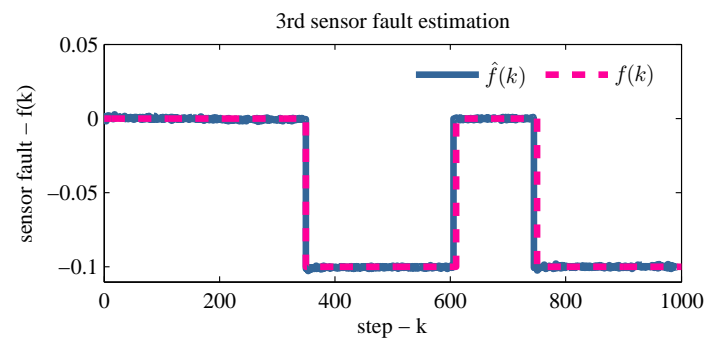

Fig. 15 Estimation of the intermittent sensor fault - the third sensor
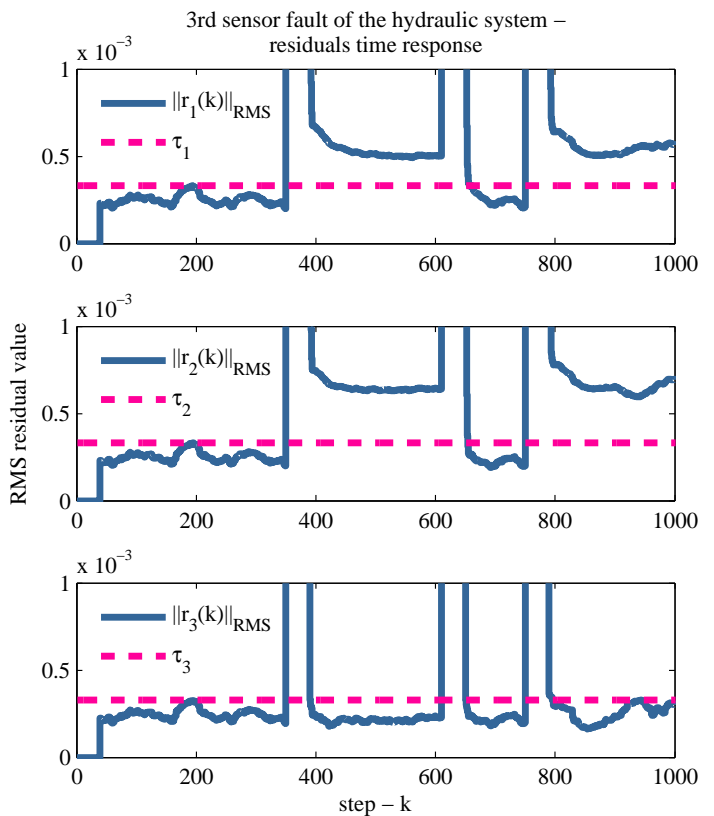

Fig. 16 Time responses of the residuals RMS values for the intermittent sensor fault - the third sensor

The results of the experiments show that the first sensor fault isolation has the longest duration. The estimated value of the each sensor fault is close to the real value when the transient processes end. The realized experiments present functionality and performance of the created sensors fault diagnosis system.

\section{CONCLUSION}

This article presents fault diagnosis algorithms design for the detection, isolation and estimation of the sensors fault. Designed fault diagnosis algorithms are implemented into the Matlab/Simulink and they are verified using simulation model of the hydraulic system. Also, the article presents methodology for the creation of the diagnosis system, which is based on the designed fault diagnosis algorithms. In fact, the diagnosis system is based on the group of the three fault estimation filters. Each fault estimation filter is able to estimate hydraulic system states and one sensor fault using principles of the Kalman filtering. The results of the simulation experiments show that the created diagnosis system can be used for the detection, isolation and estimation of sensors fault. The presented diagnosis system can isolate and estimate only the fault of one of the hydraulic system sensors, what is the disadvantage of this approach. On the other hand, this diagnosis system can be used for the time varying systems.

In respect to the goals of the project "University Science Park TECHNICOM for Innovation Applications Supported by Knowledge Technology" with subactivity "Center for Nondestructive Diagnostics of Technological Processes," it is planed to extend the fault diagnosis system to actuators fault detection, isolation and estimation ability. Also, the residual evaluation implementation using stastistical methods is one of the future research goals. 
The laboratory model of the hydraulic system (http://kyb.fei.tuke.sk/laben/modely/hyd.php) can be used for the fault diagnosis algorithms verification. Mathematical model of the mentioned hydraulic system was derived based on methods of the analytical or experimental identification and used for the various optimal control algorithms design [10], [11]. The achieved results in the modelling and control fields are the motivation for the diagnosis system creation of the laboratory model of the hydraulic system according to the methodology, which is presented in this article. Also, the control algorithms of this laboratory model can be improved to the fault tolerant form.

\section{ACKNOWLEDGEMENT}

This publication is the result of the Project implementation: University Science Park TECHNICOM for Innovation Applications Supported by Knowledge Technology - II. phase, ITMS: 313011D232 (50\%) and "Centre of Information and Communication Technologies for Knowledge Systems", ITMS: 26220120020 (20\%), both supported by the Operational Programme Research \& Development funded by the ERDF, grant TUKE FEI-2015-33: Research Laboratory of Nonlinear Underactuated Systems (30\%).

\section{REFERENCES}

[1] NOURA, H. - THEILLIOL, D. - PONSART, J. CHAMSEDDINE, A.: Fault-tolerant control systems: Design and practical applications, Springer Science \& Business Media, 2009, ISBN 978-1-84882-653-3.

[2] DING, S.: Model-based fault diagnosis techniques: design schemes, algorithms, and tools, Springer Science \& Business Media, 2008, ISBN 978-3-54076303-1.

[3] THEILLIOL, D. - NOURA, H. - PONSART, J.: Fault diagnosis and accommodation of a three-tank system based on analytical redundancy, ISA transactions, Vol. 41, No. 3, 2002, pp. 365-382, ISSN 00190578 .

[4] ZHEN-HUA, W. - RODRIGUES, M. - THEILLIOL, D. - SHEN, Y.: Sensor fault estimation filter design for discrete-time linear time-varying systems, Acta Automatica Sinica, Vol. 40, No. 10, 2005, pp. $2364-$ 2369, ISSN 1874-1029.

[5] KOUSAR, F. - ABID, M. - KHAN, A. Q. Sensor fault detection in coupled liquid tanks system, Proceedings of the 10th International Conference Frontiers of Information Technology (FIT), 2012, pp. 318-324, ISBN 978-1-4673-4946-8.

[6] IQBAL, M. - BUTT, Q. R. - BHATTI, A. I.: Linear model based diagnostic framework of three tank system, Proceedings of the 11th WSEAS International Conference on SYSTEMS, 2007, ISBN 978-1-61804027-5.

[7] ZHEN-HUA, W. - RODRIGUES, M. - THEILLIOL, D. - SHEN, Y.: Actuator fault estimation observer design for discrete-time linear parameter-varying descriptor systems, International Journal of Adaptive
Control and Signal Processing, Vol. 29, No. 2, 2015, pp. 242-258, ISSN 1099-1115.

[8] ZHEN-HUA, W. - SHEN, Y. - ZHANG, X.: Attitude sensor fault diagnosis based on Kalman filter of discrete-time descriptor system, Journal of Systems Engineering and Electronics, Vol. 23, No. 6, 2012, pp. 914-920, ISSN 1004-4132.

[9] JAJČIŠIN, Š. - JADLOVSKÁ, A.: Laboratory model of Hydraulic System Control, Electroscope - online journal for Electrotechnics, Vol. 3, No. 11, 2011 (in Slovak), ISSN 1802-4564.

[10] JAJČIŠIN, Š. - JADLOVSKÁ, A.: Generalized Predictive Control Design for a Nonlinear Hydraulic System, Acta Electrotechnica et Informatica, Vol. 11, No. 2, 2011, pp. 26-32, ISSN 1335-8243.

[11] ČERKALA, J. - JADLOVSKÁ, A.: Methodology for experimental identification of the laboratory hydraulic system, Annals of Faculty Engineering Hunedoara-International Journal of Engineering, Vol. 12, No. 3, 2014, pp. 33-40, ISSN 1584-2673.

Received February 22, 2017, accepted May 2, 2017

\section{BIOGRAPHIES}

Matej Oravec was born in March 3rd, 1991. In 2014 he graduated (MSc.) with distinction at the Department of Cybernetics and Artificial Intelligence of the Faculty of Electrical Engineering and Informatics at Technical University of Košice. Since September 2014 he has been internal PhD. student at the Department of Cybernetics and Artificial Intelligence. The topic of his dissertation thesis is focused on methods and approaches of the non-destructive diagnosis of the nonlinear dynamic systems. In addition, the sphere of his interest is physical dynamic system modelling, optimal control algorithms design and their verification on simulation and real laboratory models by Matlab/Simulink environment.

Anna Jadlovská was born in October 29th, 1960. She received her MSc. degree in the field of Technical Cybernetics at the Faculty of Electrical Engineering of the Technical University of Košice in 1984. She defended her PhD thesis in the domain of Automatization and Control in 2001 at the same University; her thesis title was "Modelling and Control of Non-linear Processes Using Neural Networks". Since 1993 she worked in the Department of Cybernetics and Artificial Intelligence Faculty of Electrical Engineering and Informatics Technical University of Košice as an Associate Assistant and since 2004 she has been working as an Associate Professor. Her main research activities include the problems of adaptive and optimal control - in particular predictive control with constraints for non-linear processes using neural networks and methods of artificial intelligence (Intelligent Control Design). She is the author of scientific articles and contributions to various journals and international conference proceedings, as well as being the co-author of some monographs. 